\title{
Making Existing Clusterings Fairer: Algorithms, Complexity Results and Insights
}

\author{
Ian Davidson, ${ }^{1}$ S. S. Ravi ${ }^{2}$ \\ ${ }^{1}$ Computer Science Department, University of California, Davis \\ ${ }^{2}$ Biocomplexity Institute \& Initiative, University of Virginia and \\ Computer Science Department, University at Albany - SUNY \\ davidson@cs.ucdavis.edu, ssravi0@gmail.com
}

\begin{abstract}
We explore the area of fairness in clustering from the different perspective of modifying clusterings from existing algorithms to make them fairer whilst retaining their quality. We formulate the minimal cluster modification for fairness (MCMF) problem where the input is a given partitional clustering and the goal is to minimally change it so that the clustering is still of good quality and fairer. We show using an intricate case analysis that for a single protected variable, the problem is efficiently solvable (i.e., in the class $\mathbf{P}$ ) by proving that the constraint matrix for an integer linear programming (ILP) formulation is totally unimodular (TU). Interestingly, we show that even for a single protected variable, the addition of simple pairwise guidance (to say ensure individual level fairness) makes the MCMF problem computationally intractable (i.e., NP-hard). Experimental results on Twitter, Census and NYT data sets show that our methods can modify existing clusterings for data sets in excess of 100,000 instances within minutes on laptops and find as fair but higher quality clusterings than fair by design clustering algorithms.
\end{abstract}

\section{Introduction}

Existing work on clustering and fairness takes a known clustering algorithm and modifies it produce fair results. The seminal work of Chierichetti et al. (2017) looked at $k$-center and $k$-median style algorithms whilst later work has explored other formulations such as spectral clustering (Kleindessner et al. 2019). However, there is a plethora of different clustering algorithms, with this decade old survey (Xu and Wunsch 2005) listing over 15 popular partitional clustering algorithms with a variety of settings, formulations and followings by end user communities. It is unlikely that fair versions of all these algorithms or new clustering algorithms will be developed. Furthermore, often clusterings results are already deployed. In these circumstances, one research direction is to modify an existing clustering to make it fairer whilst not unduly changing its quality. This paper considers this precise situation, where one already has a good clustering $\Pi$ and the goal is to modify $\Pi$ to improve its fairness with respect to a set $P$ of protected variables. We focus on the simplest but most

Copyright (c) 2020, Association for the Advancement of Artificial Intelligence (www.aaai.org). All rights reserved. common type of protected variables, namely binary variables such as gender.

A Flexible Efficient Approach To Ensure Fairness. Our approach places upper and lower bounds on the number of protected status individuals in each cluster which can be any constant expression and these bounds may even be different for each cluster. For example, if a data set $D$ has $N$ special items and $D$ has been partitioned into $k$ clusters, then the number of special data items per cluster could be set to be approximately $\frac{N}{k}$, effectively balancing the protected status instances uniformly across all clusters. Alternatively, we could require each cluster to have approximately $\frac{N \cdot\left|C_{i}\right|}{|D|}$ (where $\left|C_{i}\right|$ is the size of cluster $i$ ) protected status individuals, this would require that the ratio of the number of special items to the size of the cluster be (approximately) the same for all clusters. We now provide two fairness definitions. The first is useful to prove total unimodularity (TU) but in practice the second is more pragmatic and also gives rise to a TU constraint matrix (see Theorem 3.2):

Definition 1.1. Let $D$ be a dataset where each data item has a single binary protected attribute $x$. Let $N_{x}$ denote the number of special data items in $D$. A partition of $D$ into $k \geq 2$ is strongly fair with respect to $x$ if in each cluster, the number of special items is either $\left\lfloor N_{x} / k\right\rfloor$ or $\left\lceil N_{x} / k\right\rceil$.

This is useful in our intractability results and algorithm design. However, it is a strong requirement; hence we define a relaxed notion called $\alpha$-fairness as follows:

Definition 1.2. Consider a dataset $D$ where each data item has a single binary protected attribute $x$. Let $D_{x} \subseteq D$ denote the subset of special data items and let $N_{x}=\left|D_{x}\right|$. Let $\alpha$ be a positive integer $<\left\lceil N_{x} / k\right\rceil$. A partition of $D$ into $k \geq 2$ clusters is $\alpha$-fair wrt. $x$, if in each cluster, the number of elements from $D_{x}$ is in the range $\left[\left\lceil N_{x} / k\right\rceil-\alpha . .\left\lceil N_{x} / k\right\rceil+\alpha\right]$.

ILP Formulations for the Minimal Cluster Modification for Fairness (MCMF) Problem. A natural way to formulate the MCMF problem is as a discrete optimization problem where the goal is to minimize the effect of modifying the clusters (see Section 3 for a precise formulation). Here, the effect can be in terms of the number of instances moved or even changes in the quality of the clustering. While solving an ILP is, in general, computationally intractable, for 
our formulation of the MCMF problem, we show that (see Theorem 3.2) the constraint matrix our fairness requirements introduce are totally unimodular (TU) (Schrijver 1998). This proof involves an intricate case analysis so is presented in (Davidson and Ravi 2019). As a consequence, one can use any polynomial time linear programming (LP) algorithm to obtain integer solutions to MCMF.

\section{Summary of Main contributions.}

(1) We define a novel minimal modification problem to produce fair clustering which can post-process the results of any partitional clustering method to make them fairer whilst ensuring their clustering quality is not affected significantly. This is different from existing work that attempts to take an existing clustering algorithm and produce a fair variant of it. (2) We formulate the MCMF problem as an ILP and show that under our definitions of fairness, the formulation produces a constraint matrix that is totally unimodular (see Section 3). This constraint matrix can be paired with a variety of objectives to ensure clustering quality is not affected significantly (see Table 1). This leads to algorithms capable of modifying clusterings with millions of instances since there are well known LP solvers that run in polynomial time. Some variations to ensure fairness such as allowing overlapping clusters also have the TU property (see Section 4).

(3) If even larger clusterings are studied, in (Davidson and Ravi 2019) we show an algorithm whose worst-case run time is better than an LP solver (see Section 3).

(4) Interestingly, we show that though finding a feasible strong fair clustering (an example of group level fairness (Barocas and Selbst 2016)) is in $\mathbf{P}$ or finding a feasible clustering to satisfy commonly using popular must-link constraints (Basu, Davidson, and Wagstaff 2008) which can encode individual level fairness (Barocas and Selbst 2016) is in $\mathbf{P}$, finding a feasible clustering to satisfy both requirements is computationally intractable (see Section 5, Theorem 5.1).

(5) Experimental results show that our method is computationally efficient (as expected) and our objectives useful for post-processing the results from k-means, k-medians and spectral clustering algorithms. The experiments are for Census/Adult (48K), NYT (300K) and Twitter Healthcare (58K) datasets. We show that our method of post-processing can produce similar fairness results as jointly finding a good but fair clustering (Chierichetti et al. 2017).

Organization. We begin by discussing related work in section Section 2. We then formulate the minimal clustering modification for fairness (MCMF) problem in Section 3 as an ILP and show that its constraint matrix is totally unimodular. We establish the complexity of achieving fairness while satisfying instance-level constraints in Section 5. We then present results from our experiments in Section 6. Conclusions and directions for future work are provided in Section 7. Reference (Davidson and Ravi 2019) contains a linear time algorithm the the MCMF problem.

\section{Related Work}

We briefly review two related work areas, namely fairness in machine learning (ML) and minimal modification of clustering. Fairness in ML is an emerging area that has received much attention in the context of supervised learning, often under different names such as algorithmic bias (Thanh, Ruggieri, and Turini 2011). More recently, in clustering (i.e., unsupervised learning), the issue of fairness generally aims at balancing protected individuals across clusters as we have.

All of the work below uses a similar fairness measure (shown below) and focuses on simple $k$ means/medians/centers algorithms with the exception of (Kleindessner et al. 2019) which explores spectral clustering. The idea of balancing protected individuals aims to address the disparate impact doctrine (Feldman et al. 2015; Friedler, Scheidegger, and Venkatasubramanian 2016) and was formalized in the seminal work of Chierichetti et al. (2017). They assumed that each object has one of two colors (red or blue). Letting the number of instances of each type in cluster $i$ be $R_{i}$ and $B_{i}$ respectively, the fairness of a clustering is $\min \left(\min \left[\frac{R_{1}}{B_{1}}, \frac{B_{1}}{R_{1}}\right], \ldots, \min \left[\frac{R_{k}}{B_{k}}, \frac{B_{k}}{R_{k}}\right]\right)$. Their work creates fairlets (groups of instances) which when post processed by $k$-center and $k$-medians are guaranteed to produce a specified level of fairness and achieve a constant factor approximation with respect to cluster quality. As seen in Table 4, by ensuring that cluster sizes are not unduly changed, the Chierichetti et al. (2017) measure of fairness and our measure can yield similar results. However, a stronger statement regarding their equivalence is left to future work.

The work of Backurs et al. (2019) showed how a fairlet decomposition algorithm can be implemented to run in nearly linear time. The work of Rösner and Schmidt (2018) extended the work of (Chierichetti et al. 2017) by allowing objects with more than two colors (i.e., three or more protected attributes) but assume that each object has only one color. Bera et al. (2019) also consider three or more colors and allow an object to have more than one color. They also allow users to specify upper and lower bounds on fairness measures for each cluster and develop clustering algorithms under any $\ell_{p}$ norm.

In this paper we take an alternative path of improving fairness by post-processing (i.e., by minimal modification of) clustering results produced by existing methods. The idea of minimal modification of clustering solutions has been explored before by ourselves (Kuo et al. 2017); however, that focus is on human-in-the-loop style settings where the domain expert can choose to adjust geometric properties of the cluster such as diameter. Hence, the theorems and results these two papers are fundamentally different. Further, the focus of this earlier work was on improving cluster quality by moving a small number of instances between clusters; it did not take fairness into consideration. Further, due to the use of the constraint programming, this earlier work scales only to data sets of size at most 1000 . In contrast, our modification algorithm for improving fairness scales to very large data sets (hundreds of thousands of points) even on a laptop (see Section 6).

\section{An ILP Formulation and Proof of Total Unimodularity}

Here we show an ILP formulation of the MCMF problem which can find fairer clusters. The reader may also refer to (Davidson and Ravi 2019) for implementation details. 


\begin{tabular}{|l|l|}
\hline Measure & $\begin{array}{l}\text { Meaning of Objective } \\
\text { Function }\end{array}$ \\
\hline \hline $\mathbf{w}=\mathbf{1}$ & $\begin{array}{l}\text { The number of instances } \\
\text { moved. }\end{array}$ \\
\hline $\begin{array}{l}w_{i}=\sum_{j} \frac{d\left(i, C_{j}\right)}{k}- \\
d\left(i, C^{*}\right)\end{array}$ & $\begin{array}{l}\text { The increase in mean L2 } \\
\text { (distortion) or L1 (me- } \\
\text { dian) distances (average dis- } \\
\text { tance from } i \text { to cluster } j: \\
\left.d\left(i, C_{j}\right)\right) .\end{array}$ \\
\hline$w_{i}=\sum_{j} E(i, j):(i, j) \in$ & $\begin{array}{l}\text { The increase in external de- } \\
\text { gree by moving instance } i \\
\text { away from cluster } \Pi_{a} .\end{array}$ \\
\hline
\end{tabular}

Table 1: Several penalty schemes and their meaning when used in Equation (1). Each measures the increase if $i$ is moved away from the cluster $\left(C^{*}\right)$ to which it is assigned in $Z^{*}$. We assume that $Z^{*}$ is optimal for the given objective.

We then show the resultant constraint matrix is totally unimodular (TU) and hence the ILP is efficiently solvable. Our formulation can be used to ensure any upper and lower bound on the number of protected variables in a cluster. These bounds need not be the same for each cluster and most importantly, since TU depends on the constraint matrix coefficients and not on the right-hand side of the constraints, these formulations are also efficiently solvable.

Our aim is the following: given a desirable existing clustering (defined in an $k \times n$ allocation matrix $Z^{*}$ ), find a minimal modification that makes the clustering fairer with respected to the single protected variable $P$. In our experiments, we consider a formulation to allow many protected variables. We also discuss other settings which lead to TU constraint matrices.

Objective. We wish to find another allocation matrix $Z$ that is fairer but similar to $Z^{*}$. As $z_{i}^{*}$ and $z_{i}$ are both binary column vectors indicating what single cluster the $i^{\text {th }}$ instance belongs to, $\sum_{i}\left(z_{i}^{*}\right)^{T} \times\left(z_{i}\right)$ counts the number of agreements between $Z$ and $Z^{*}$ which forms the basis of useful objectives. We can easily encode preferences/importance amongst instances by having a penalty $\left(w_{i}\right)$ (see Equation (1)) if instance $i$ is moved which can take on a variety of semantic meanings. See Table 1 for some examples. The first simply minimizes the number of instances moved, the second is useful for centroid based methods such as $k$-means since it minimizes the increase in distortion (assuming that the existing solution minimizes the distortion) and the last is useful for graph based formulations since it minimizes increase in cut cost (again assuming that the existing solution minimizes the mincut). Of course, domain experts can easily encode their own preference schemes.

$$
\operatorname{argmin}_{Z} \sum_{i} w_{i}\left[1-\left(z_{i}^{*}\right)^{T} \times\left(z_{i}\right)\right]
$$

Adding Constraints With Slack Variables. The aim of the constraints are two-fold, to balance the protected variable whilst also restricting $Z$ to be a legal cluster allocation matrix. Note that we use the encoding where indicator vectors are stacked column-wise, that is $z_{i, j}=1$ iff instance $j$ is assigned to cluster $i$. We encoded the protected status as a vector $P$ of length $n$ with an entry of 1 if the instance has the status otherwise 0 . Our first two constraints require that the distribution of the protected variable be upper and lower bounded. For example, to follow our definition of strict fairness (see Definition 1.1) we would have the constraint $\left\lfloor\frac{|P|}{k}\right\rfloor \leq \sum_{j} p_{i} z_{i, j} \leq\left\lceil\frac{|P|}{k}\right\rceil \forall i$ with $u$ (upper) and $l$ (lower) being the slack variables. In the following equation we generalize this to any upper and lower bounds and note they can vary depending on the cluster. (We use $|P|$ to denote the number of non-zero entries in the vector $P$.) The last set of constraints below (i.e., $\sum_{i} z_{i, j}=1 \forall j$ ) simply require that $Z$ is a valid allocation matrix. Note again that the instances are stacked column-wise in $Z$.

$$
\begin{gathered}
\sum_{j} p_{j} z_{i, j}+u_{i}=U_{i}, \quad \forall i \\
-\sum_{j} p_{j} . z_{i, j}+l_{i}=-L_{i}, \quad \forall i \\
\sum_{i} z_{i, j}=1, \quad \forall j
\end{gathered}
$$

Note that when $U_{i}=\lceil|P| / k\rceil$ and $L_{i}=\lfloor|P| / k\rfloor$, for $1 \leq i \leq k$, Lemma 3.2 points out that there is always a solution to the above set of constraints. We will make a similar observation regarding a relaxed version of the fairness requirement later.

Total Unimodularity of Constraint Matrix. It is well known (Schrijver 1998) that if the constraint matrix of an ILP is totally unimodular (TU) then we can solve the problem using an $L P$ (linear program) solver and the solution will still be integral. Further, linear programming problems can be solved in $O\left(n(n+d)^{1.5} L\right)$ time, where $n$ is the number of variables, $d$ is the number of constraints and $L$ is the total number of bits needed to encode all the constants specified in the LP (Vaidya 1989). This running time is clearly polynomial in the input size.

In the above equations, there are $k n$ regular variables (namely, $z_{11}, z_{12}, \ldots, z_{1 n}, \ldots, z_{k 1}, z_{k 2}, \ldots, z_{k n}$ ) and $2 k$ slack variables (namely $u_{1}, \ldots, u_{k}$ and $l_{1}, \ldots, l_{k}$ ). For the purpose of constructing the constraint matrix $C$, we will use the following order of these $k n+2 k$ variables: $\left\langle z_{11}, z_{12}, \ldots\right.$, $\left.z_{1 n}, \ldots, z_{k 1}, z_{k 2}, \ldots, z_{k n}, u_{1}, \ldots, u_{k}, l_{1}, \ldots, l_{k}\right\rangle$. Matrix $C$ has $2 k+n$ rows (one corresponding to each constraint) and $n k+2 k$ columns (one corresponding to each variable). In $C$, we will list the $2 k$ constraints corresponding to Equations (2) and (3) in the order specified by those equations. This is followed by the $n$ constraints in the order specified by Equation (4). Note that each entry of $C$ is from $\{-1,0,+1\}$. In each row of $C$ (which specifies one constraint), we will list the coefficients of the $k n+2 k$ variables in the order specified above. We refer to the first $k n$ columns of $C$ as regular variable columns and the last $2 k$ columns as slack variable columns. Using this terminology, we can prove the following lemma.

Lemma 3.1. (a) In any regular variable column of $C$, there are at most three non-zero elements. (b) In any slack variable 
column of $C$, there is exactly one element with value 1 ; the other entries in that column are 0.

Proof: See (Davidson and Ravi 2019).

To prove the TU property of the constraint matrix $C$, we will use the following result, which is Theorem 19.3 in (Schrijver 1998).

Theorem 3.1. TU Identity (Schrijver 1998) Let $C$ be a matrix such that all its entries are from $\{0,+1,-1\}$. Then $C$ is totally unimodular, i.e., each square submatrix of $C$ has determinant $0,+1$, or -1 if every subset of rows of $C$ can be split into two parts $A$ and $B$ so that the sum of the rows in $A$ minus the sum of the rows in $B$ produces a vector all of whose entries are from $\{0,+1,-1\}$.

Theorem 3.2. The matrix $C$ formed by the coefficients of the constraints used to encode Equations (2) through (4) is totally unimodular.

Proof: See (Davidson and Ravi 2019).

An Alternative More Efficient Algorithm Only For Strict Fairness. It is also possible to obtain another efficient algorithm for MCMF using the following idea. Given an arbitrary distribution of the special items into $k$ clusters, we use Lemma 3.2 (below) to identify which clusters have an "excess" amount of special items and which ones are "deficient" with respect to special items. It can be seen using Lemma 3.2, the total number of excess items gives the lower bound on the number of special items that must be moved to achieve strong fairness. The algorithm provides an optimal solution by ensuring that the number of special items moved between clusters is equal to the lower bound. As the details of the algorithm involve many cases, a description of the algorithm is given in (Davidson and Ravi 2019). This algorithm's worst-case running time is better than that of an LP solver.

The following result shows that there exists a distribution of special items into clusters that is a necessary and sufficient condition for a strongly fair clustering of $D$.

Lemma 3.2. [A necessary and sufficient condition for Strong Fairness.] Let $D$ be a data set with one binary protected attribute $x$. Let $D_{x} \subseteq D$ denote the subset of special data items and let $N_{x}=\left|\bar{D}_{x}\right|$. Let $q$ and $r$ be non-negative integers such that $N_{x}=q k+r$ with $k$ being the number of clusters and $0 \leq r \leq k-1$. A partition of $D$ into $k$ clusters is strongly fair with respect to $x$ if and only if it has exactly $r$ clusters each with $\left\lceil N_{x} / k\right\rceil$ special data items and $k-r$ clusters each with $\left\lfloor N_{x} / k\right\rfloor$ special data items.

Proof: See (Davidson and Ravi 2019).

\section{Other Variations that Are and Are Not TU}

Here we discuss variations some of which lead to constraint matrices with the TU property while others do not. It is important to bear in mind that the proof of TU only depends on the coefficients of the constraint matrix. It does not depend on the objective function (which is why we can have the variations such as those in Table 1); nor does it depend on the right hand side of the constraints.

Allowing Overlapping Clusters. A desirable situation to enforce fairer cluster is to allow an instance to belong to multiple $(s)$ clusters. Our slack variable formulations easily facilitates an instance belongs to at most $s$ clusters. This has the benefit of spreading the protected individuals to multiple clusters, that is we have $\sum_{i} z_{i, j}=s, \forall j$ but since this does not change the coefficients of the constraint matrix (only the constant in the equality) this formulation is also TU.

Multiple Protected Variables. When there are $r \geq 2$ protected variables, $r-1$ additional sets of constraints similar to Equations (2) and (3) must be added. Since the same data item may have many protected attributes, it is not clear whether the resulting constraint matrix satisfies the TU property. Determining if this case satisfies the TU property will be left for future work.

Weighted or Continuous Protected Variables. The proofs of TU require the constraint matrix to contain entries of only $\{-1,0,+1\}$. In general, any work that requires degrees of protection (i.e., age) even if encoded in ordinal form cannot be encoded as TU matrix to our knowledge.

\section{Difficulty of Satisfying Group and Individual Level Fairness}

Our measure of strong fairness (Definition 1.1) is a group (cluster) level measure (Barocas and Selbst 2016). An alternative measure of fairness is individual level (Barocas and Selbst 2016) where we require similar individuals to be treated/clustered the same. This can be encoded as the popular must-link (ML) constraints (Wagstaff and Cardie 2000; Basu, Davidson, and Wagstaff 2008) where the constraint $\operatorname{ML}(a, b)$ requires data items $a$ and $b$ to be in the same cluster.

As shown in Theorem 3.2 satisfying strong fairness is computationally tractable. Similarly the feasibility problem with respect to ML constraints (i.e., given a data set $D$, an integer $k$ and a set $S$ of ML constraints, can $D$ be partitioned into $k$ clusters so that all the ML constraints in $S$ are satisfied?) can also be solved efficiently (Davidson and Ravi 2007). However, we now show satisfying both requirements is computationally intractable (Theorem 5.1). We start with a definition of the corresponding feasibility problem.

Feasibility of Strongly Fair Clustering under ML Constraints (FSFC-ML)

Instance: A dataset $D$ where each item has a set of attributes, a protected attribute $x$, an integer $k \leq|D|$, a set $S$ of ML constraints.

Question: Can $D$ be partitioned into $k$ clusters so that the resulting clustering (i) is strongly fair with respect to $x$ and (ii) satisfies all the ML constraints in $S$ ?

The following result points out that FSFC-ML is computationally intractable.

Theorem 5.1. Problem FSFC-ML is NP-complete.

Proof: We use a reduction from the 3-PARTITION problem (Garey and Johnson 1979). The details are in (Davidson and Ravi 2019).

A consequence of Theorem 5.1 is that the minimum modification problem where the goal is to achieve group level fairness (as per our definition) and individual level fairness is computationally intractable. 


\section{Experimental Results}

To illustrate the usefulness of our method we explore several large data sets (Adult/Census, Twitter Healthcare and NYT) on both k-means, k-medians and spectral clustering algorithms. Since no other work attempts to post process results to make them fairer we do not present the standard "Us vs Them" tables of results but instead attempt to illustrate our work's uses, limitations and comparisons to fair-by-design clustering algorithms. We attempt to answer the following:

Q1. What is the impact of our modification approach on real world data sets? Can our objectives in Table 1 find fairer clusters whilst also retaining high quality clustering?

Q2. How does making existing clusterings fairer compare to approaches that find fair clusterings to begin with (e.g., Chierichetti et al. (2017))?

Q3. What is the approximate run time of our method and the impact of increasing the number of instances and clusters?

We begin with an illustrative data set $(\approx 50 \mathrm{k}$ instances $)$ used by many previous fairness papers and the move onto a larger collection of data sets $(\approx 58 \mathrm{~K}$ and $300 \mathrm{k}$ instances).

\subsection{Q1 - Effects of Post-Processing}

Here we first analyze the well studied Adult dataset (e.g., (Chierichetti et al. 2017; Backurs et al. 2019)) that consists of 48,842 individuals (males $66.8 \%$, females $33.2 \%$ ) from the UCI repository (Dheeru and Karra Taniskidou 2017).

Case Study: Post-Processing Results of k-Means. The best clustering result of partitioning this data into 5 clusters using $k$-means is shown in Table 2 . We immediately see that the first two clusters are desirable from a marketing perspective as they consist of highly educated individuals with high gains (related to income) who can be targeted for better loans, credit cards, ads etc. to them. However, they are overwhelmingly male, with no more than $21 \%$ of the total population per cluster being female. Note the proportion of females in this data set is $33.2 \%$.

To make these first two clusters fairer we apply our method by placing bounds on the first and second cluster's protected status ratios to be $0.5 \pm 0.05$ with the remaining clusters' proportion of females to be their current values as reported in Table $2 \pm 0.15$. This is achieved by setting the $U_{i}$ and $L_{i}$ bounds in Equations (2) and (3). We then applied the minimal modification method with the second objective in Table 1 as it is compatible with the $k$-means objective. The results are shown in Table 3. Since we used $k$-means clustering we measure the impact of our modified clustering in terms of the increase of the distortion (the objective function used by $k$-means). We found that female instances from Cluster 4 were placed in Cluster 1 and Cluster 2.

The results show several interesting insights:

1. We find that when only modifying to make clusters fairer, the distortion only increased by $2 \%$. This indicates our objective function in Table 1 is useful at ensuring the clustering quality is not diminished.

2. However, the description and sizes of the clusters do change (highlighted by bold in Table 3 ) sometimes adversely. For example, the second cluster now becomes less desirable from a marketing perspective as it contains less educated

\begin{tabular}{|c|c|c|c|c|c|}
\hline Cluster & 1 & 2 & 3 & 4 & 5 \\
\hline Female & $21 \%$ & $12 \%$ & $25 \%$ & $51 \%$ & $14 \%$ \\
\hline Size & 5352 & 2776 & 15180 & 20182 & 5352 \\
\hline age & & 47 & 43 & 31 & 46 \\
\hline educ. & Bachelors & Bachelors & HS-grad & Some- & Some- \\
\hline status & Married & Married & Married & Never & Married \\
\hline occup. & Prof & Sales & Craft & Prof & Exec \\
\hline gain & 3910 & 2887 & 353 & 233 & 2556 \\
\hline
\end{tabular}

Table 2: For $k$-means and census dataset. A description of the best clustering found using $k=5$ (minimized distortion over 1000 random restarts) and the fraction of the protected variable (females) per cluster. The distortion of the solution is 110402.48 . This is the given clustering we shall minimally modify to obtain results in Tables 3 and 4 .

\begin{tabular}{|c|c|c|c|c|c|}
\hline Cluster & 1 & 2 & 3 & 4 & 5 \\
\hline Female & $45 \%$ & $45 \%$ & $34 \%$ & $25 \%$ & $28 \%$ \\
\hline Size & 5923 & 6321 & 10231 & 14001 & 12366 \\
\hline age & 41 & 43 & 44 & 35 & 45 \\
\hline educ. & Bachelors & $\begin{array}{l}\text { HS- } \\
\text { grad }\end{array}$ & HS-grad & $\begin{array}{l}\text { Some- } \\
\text { college }\end{array}$ & Bachelors \\
\hline status & Married & Never & Married & Never & Married \\
\hline occup. & Prof & Sales & Craft & Prof & Sales \\
\hline gain & 2834 & 2532 & 1431 & 452 & 2641 \\
\hline
\end{tabular}

Table 3: For $k$-means, census dataset and our method. A description of the clusters found using our method (using second objective in Table 1) by minimally modifying the clustering described in in Table 2. The distortion of this solution increased approximately $2 \%$ to 112400.68 . Compare with Table 2 . Interesting changes between that table are bolded.

\begin{tabular}{|l|l|l|}
\hline Attribute Focus & $\begin{array}{l}\text { Distortion } \\
\text { Increase }\end{array}$ & $\begin{array}{l}\text { Fairness } \\
\text { Decrease per } \\
\text { (Chierichetti et al. 2017) }\end{array}$ \\
\hline Education & $2.1 \%$ & $1.3 \%$ \\
\hline Marital Status & $1.8 \%$ & $2.5 \%$ \\
\hline $\begin{array}{l}\text { Education + } \\
\text { Marital Status }\end{array}$ & $8.0 \%$ & $3.6 \%$ \\
\hline Keep Cluster Sizes $\pm 0.05 \%$ & $15.4 \%$ & $0.1 \%$ \\
\hline $\begin{array}{l}\text { Education + Keep Cluster } \\
\text { Sizes } \pm 0.05 \%\end{array}$ & $19.8 \%$ & $0.8 \%$ \\
\hline $\begin{array}{l}\text { Marital Status + Keep Cluster } \\
\text { Sizes } \pm 0.05 \%\end{array}$ & $20.3 \%$ & $0.9 \%$ \\
\hline $\begin{array}{l}\text { Education + Marital Status + } \\
\text { Keep Cluster Sizes } \pm 0.05 \%\end{array}$ & $23.6 \%$ & $0.8 \%$ \\
\hline
\end{tabular}

Table 4: $k$-means, census dataset and our method with multiple constraints on variables. The distortion increase of the modified to be fairer (for gender) clustering over the clustering in Table 2 but we now require other properties in Table 2 to be retained. 
individuals who are not married. This motivates our next experiments on multiple protected attributes.

Experiments with multiple protected attributes to overcome challenges. The last item in the above list is a challenge with just balancing a single protected variable. To address this, we can constrain other variables, even though they are not protected. It is important to realize that we can constrain the size of the clusters by creating a dummy protected variable that every instance possesses. Thus to better ensure fairness (wrt to gender) across the clusters whilst retaining other properties of the two desirable clusters we also constrain education, marital-status. The increase in distortion for these more complex experiments is shown in Table 4. Not surprisingly, the requirement of keeping cluster sizes similar to their previous values produces a greater increase in distortion. Next we measure the fairness of our clusterings using the classic fairness measure of (Chierichetti et al. 2017). As expected we find (Table 4 last column) no large difference as both measures are based on cardinality. In question Q2 we explore whether the two methods produce different results.

More Data Sets and Experiments With $k$-Means and Spectral Clustering. We now explore two larger data sets: (i) the NYT Articles Bags of Words Data Set (300,000 instances) and (ii) Twitter Data of Health News (58,000 instances). Each data set is represented by the 1000 most frequent words including gender (male, female), race (black, hispanic, white) and age (elderly, young). The former data set is already processed whilst we processed the latter using the BOW toolkit (https://www.cs.cmu.edu/ mccallum/bow/). For each data set we find the best $k=10$ clustering using plain $k$-means and spectral clustering $+k$-means (both from 1000 random restarts). We then reported the increase in distortion and cut cost by ensuring fairness across all clusters for a variety of key words mentioned in Table 5 and Table 6. For spectral clustering (von Luxburg 2006) we created a fully connected graph based on the cosine distance between bags of words vectors and then created a spectral embedding into 10 dimensional space and used $k$-means to find 10 clusters. We used our third objective function in Table 1 which is in principle similar to the spectral clustering objective function (from a graph cut perspective); see Tables 5 and 6.

As before we found that modifying a clustering to ensure fairness for a single protected attribute can be achieved by minimally increasing the objective function of the algorithm. However, balancing multiple protected attributes produces a greater increase than for the sum of the increase for the same two variables. For example in Table 5 balancing Female and $\mathrm{Black}$ produced a distortion increase of $5.9 \%$ but just Female or just Black produces increases of $1.3 \%$ and $1.9 \%$ respectively.

\subsection{Q2 - Direct Fair Clustering Comparison}

Here we answer the important question of how postprocessing an existing clustering to make it fairer compares to finding fair clusters to begin with. In Table 4 we showed that the classic measure of fairness (Chierichetti et al. 2017) (see section 2) is similar to our own as they are both cardinality based. However, this is different from the question

\begin{tabular}{|l|l|l|}
\hline Word Focus & $\begin{array}{l}\text { Distortion } \\
\text { Increase }\end{array}$ & $\begin{array}{l}\text { Cut Cost } \\
\text { Increase }\end{array}$ \\
\hline Base Clustering Method & 0 & 0 \\
\hline Female & $1.3 \%$ & $1.8 \%$ \\
\hline Black & $1.9 \%$ & $2.3 \%$ \\
\hline Elderly & $2.3 \%$ & $3.1 \%$ \\
\hline Female, Black & $5.9 \%$ & $7.2 \%$ \\
\hline Female, Elderly & $6.8 \%$ & $8.3 \%$ \\
\hline Black, Elderly & $7.1 \%$ & $8.9 \%$ \\
\hline Female, Black, Elderly & $13.9 \%$ & $17.3 \%$ \\
\hline Female + Cluster Sizes $\pm 5 \%$ & $18.1 \%$ & $20.3 \%$ \\
\hline
\end{tabular}

Table 5: k-means, spectral clustering and our method for NYT data set. The increase in distortion if we minimally modify the clustering of the NYT Articles Bag of Words Data Set with 10 groups using $k$-means and spectral clustering. Each row shows the increase in distortion and cut-cost caused by a fairness requirement.

of does post-processing to increase fairness find the same, better or worse clusterings as attempting to find a fair clustering to begin with. To explore this question, we used the scalable version of (Chierichetti et al. 2017), that is, the work of (Backurs et al. 2019) which implements fair k-medians. The work on fair spectral clustering (Kleindessner, Awasthi, and Morgenstern 2019) could be a suitable comparison but for our data sets of $48 \mathrm{k}, 58 \mathrm{k}$ and $300 \mathrm{k}$ instances it was not scalable as the resultant affinity matrices were nearly $300 \mathrm{~Gb}$ large (i.e., to encode a 300k x 300k matrix of short integers).

We performed two experiments. Firstly, we ran both methods (k-medians ${ }^{1}$ plus ours (objective function 2 with L2 distance in Table 1) and (Backurs et al. 2019) for k-medians) on the same collection of bootstrapped samples ( $50 \%$ of the original data set size) for our three data sets and measured the normalized Rand Index between the clusterings found by the two methods. If the Rand Index were 1 the clusterings found are identical. Table 7 (2nd column) shows our methods do not find the exact same clustering. However, if we post-process (using our method and a constraint to retain cluster sizes) the result of the (Backurs et al. 2019) method it does not unduly change the resultant clustering (column 3 Table 7).

We next explored how the output of the two methods are different. To achieve this we plot the census data experiments in a $2 \mathrm{D}$ scatter plot where one dimensions is the objective of the k-medians algorithm whilst the other is the fairness criterion used by the algorithms. Since the two notions of fairness used are similar but not identical we have two plots in Figure 1. We find that as expected each method is better at optimizing its own measure of fairness but our method is on average better at finding more compact clusters (according to the objective of k-medians). This is not unexpected as the work of (Backurs et al. 2019) guarantees fairness but has a weaker approximation bound than MATLAB's k-medians implementation.

\footnotetext{
${ }^{1}$ The theory and applied literature use different terms for the same algorithm. We use the k-medoid MATLAB algorithm which is referred to the k-medians algorithm in the theory literature.
} 


\begin{tabular}{|l|l|l|}
\hline Word Focus & $\begin{array}{l}\text { Distortion } \\
\text { Increase }\end{array}$ & $\begin{array}{l}\text { Cut Cost } \\
\text { Increase }\end{array}$ \\
\hline Base Clustering Method & 0 & 0 \\
\hline Female & $2.4 \%$ & $2.1 \%$ \\
\hline Elderly & $3.2 \%$ & $3.8 \%$ \\
\hline Female, Elderly & $7.4 \%$ & $8.8 \%$ \\
\hline Female + Cluster Sizes $\pm 5 \%$ & $17.3 \%$ & $19.4 \%$ \\
\hline
\end{tabular}

Table 6: $k$-means and spectral clustering and our method for Healthcare Data Set. The increase in distortion if we minimally modify the clustering of the Twitter Healthcare Data Set with 10 groups using $k$-means and spectral clustering. Each row shows the increase in distortion and cut-cost caused by adding a fairness requirement.

\begin{tabular}{|l|l|l|}
\hline Data Set & $\begin{array}{l}\text { Adjusted } \\
\text { RI }\end{array}$ & $\begin{array}{l}\text { Change in Fairness (num- } \\
\text { ber of instances moved) af- } \\
\text { ter post-processing results } \\
\text { from (Backurs et al. 2019). }\end{array}$ \\
\hline Adult/Census & 0.95 & $0.18 \%(0.05 \%)$ \\
\hline NYT & 0.75 & $1.1 \%(0.13 \%)$ \\
\hline Healthcare & 0.85 & $1.3 \%(0.11 \%)$ \\
\hline
\end{tabular}

Table 7: Comparison of post-processing for fairness versus searching for fair clusterings for 350 bootstrap samples each of the Census, NYT and Twitter data sets. The second column shows the Rand Index (RI) between the clustering each method finds averaged over 100 bootstrap samples. The third column shows how applying our method after finding a fair clustering decreases the fairness.
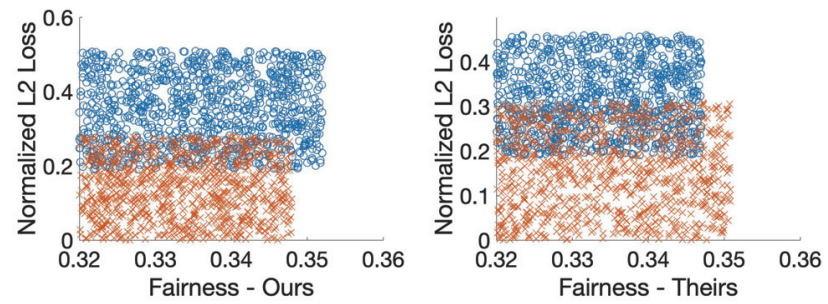

Figure 1: For 350 boostraps of the Census data set, the comparison of our method (crosses) vs Chierichetti et al. (2017) (circles) of the k-medians loss versus our measure of fairness (left) and Chierichetti et al. measure (right).

\begin{tabular}{|c|c|}
\hline $\mathbf{k}$ & run-time \\
\hline 2 & $3.8 \mathrm{~s} / 4.3 \mathrm{~s}$ \\
4 & $6.1 \mathrm{~s} / 6.5 \mathrm{~s}$ \\
8 & $8.3 \mathrm{~s} / 9.1 \mathrm{~s}$ \\
16 & $27.30 \mathrm{~s} / 23.1 \mathrm{~s}$ \\
32 & $75.43 \mathrm{~s} / 85.1 \mathrm{~s}$ \\
\hline
\end{tabular}

\begin{tabular}{|c|c|}
\hline $\mathbf{n}$ & run-time \\
\hline 1000 & $0.20 \mathrm{~s} / 0.29 \mathrm{~s}$ \\
2000 & $0.81 \mathrm{~s} / 0.95 \mathrm{~s}$ \\
4000 & $1.11 \mathrm{~s} / 1.45 \mathrm{~s}$ \\
8000 & $3.23 \mathrm{~s} / 4.93 \mathrm{~s}$ \\
16000 & $16.41 \mathrm{~s} / 18.55 \mathrm{~s}$ \\
32000 & $69.32 \mathrm{~s} / 73.81 \mathrm{~s}$ \\
\hline
\end{tabular}

Table 8: Scalability and NYT (left) and Twitter Health care (right) data sets. The mean run time over 100 experiments on a single core of a MacBook Pro laptop (i5 processor) for a randomly created subset of the data sets. Left: 10,000 instances data set and varying numbers of clusters. Right: 5 clusters and varying sized data set.

\subsection{Q3 - Scalability}

The TU proof ensures that an ILP formulation can be solved by an LP solver, but this can still take polynomial time. For our previous data sets we found that instances of the Adult data set took under one minute to run on a single core of a MacBook laptop. For the larger NYT data set time was under 5 minutes and for the Twitter data set it was 4 minutes. Here we wish to see how the run time of our algorithm is affected by increasing the number of clusters and number of instances. We explore the laptop run time for the NYT and Twitter Healthcare data sets in Table 8 of various samples. We averaged results over 100 experiments with 25 experiments each balancing Female, Black, Elderly and Hispanic to match the population ratios.

\section{Discussion and Conclusions}

We explored the novel idea of post-processing the results of existing clustering algorithms to make them fairer. We formulated the problem as an ILP and showed using an intricate case analysis that the resultant constraint matrix is totally unimodular (TU). This means that we can solve the ILP using an LP solver and thus obtain a polynomial time algorithm. We showed some variations such as a relaxed condition for fairness, overlapping clusters and importance penalty functions also have TU constraint matrices. However, the TU requirement means that interesting settings such as continuous protected variables may not be efficiently solvable.

Our complexity results showed an interesting conundrum. Though finding a strictly fair clustering for a single protected status variable (a type of group level fairness) is tractable and though finding a clustering to satisfy popular must-link constraints (which can encode individual-level fairness) is also tractable, satisfying both is intractable.

Our experiments aimed to shed light on the strengths and limitations of the approach and the general problem of making clusterings fairer. We found that though we were able to improve the fairness of large data sets efficiently on standard laptops some as big as $300 \mathrm{~K}$ instances in 5 minutes or under, we observed several interesting phenomena when attempting to find fair clusters. Firstly, making existing clusterings fair for a single protected variable can be achieved with minimal decrease in the clustering quality for a variety of clusterings produced by fundamentally different algorithms (k-means and spectral clustering). But this could have the effect of unduly influencing the composition of the clustering (e.g., cluster 2 in Table 3). We showed how this could be addressed by using our formulation to balance multiple variables (even though they are not protected) including the cluster sizes. However, balancing multiple protected variables can decrease the cluster quality substantially. We show that our measure of fairness does not produce fundamentally different results than that of the seminal work in the field (Chierichetti et al. 2017) by showing (for example) that post-processing the results of their output minimally changes the clustering. However, our method does have the benefit of not being tied to a particular clustering algorithm and is scalable due to our TU result.

Acknowledgments: We thank the referees for helpful comments. This work was supported in part by NSF Grants IIS- 
1908530 and IIS-1910306 titled: "Explaining Unsupervised Learning: Combinatorial Optimization Formulations, Methods and Applications".

\section{References}

Backurs, A.; Indyk, P.; Onak, K.; Schieber, B.; Vakilian, A.; and Wagner, T. 2019. Scalable fair clustering. To Appear in ICML.

Barocas, S., and Selbst, A. D. 2016. Big data's disparate impact. California Law Review 671:671-732.

Basu, S.; Davidson, I.; and Wagstaff, K. 2008. Constrained clustering: Advances in algorithms, theory, and applications. CRC Press.

Bera, S. K.; Chakrabarty, D.; and Negahbani, M. 2019. Fair algorithms for clustering. arXiv preprint arXiv:1901.02393.

Chierichetti, F.; Kumar, R.; Lattanzi, S.; and Vassilvitskii, S. 2017. Fair clustering through fairlets. In Proc. NeurIPS, 5036-5044.

Davidson, I., and Ravi, S. S. 2007. The complexity of non-hierarchical clustering with instance and cluster level constraints. Data Min. Knowl. Discov. 14(1):25-61.

Davidson, I., and Ravi, S. S. 2019. Making existing clusterings fairer: Algorithms, complexity results and insights. Technical report, University of California, Davis, CA . https://web.cs.ucdavis.edu/ davidson/Publications/ TR_AAAI2020.pdf.

Dheeru, D., and Karra Taniskidou, E. 2017. UCI machine learning repository.

Feldman, M.; Friedler, S. A.; Moeller, J.; Scheidegger, C.; and Venkatasubramanian, S. 2015. Certifying and removing disparate impact. In Proceedings of the 21th ACM SIGKDD International Conference on Knowledge Discovery and Data Mining, Sydney, NSW, Australia, August 10-13, 2015, 259268.

Friedler, S. A.; Scheidegger, C.; and Venkatasubramanian, S. 2016. On the (im)possibility of fairness. CoRR abs/1609.07236.

Garey, M. R., and Johnson, D. S. 1979. Computers and Intractability: A Guide to the Theory of NP-completeness. San Francisco: W. H. Freeman \& Co.

Kleindessner, M.; Awasthi, P.; and Morgenstern, J. 2019. Fair k-center clustering for data summarization. To Appear in ICML.

Kleindessner, M.; Samadi, S.; Awasthi, P.; and Morgenstern, J. 2019. Guarantees for spectral clustering with fairness constraints. To Appear in ICML.

Kuo, C.-T.; Ravi, S.; Dao, T.-B.-H.; Vrain, C.; and Davidson, I. 2017. A framework for minimal clustering modification via constraint programming. In AAAI, 1389-1395.

Rösner, C., and Schmidt, M. 2018. Privacy preserving clustering with constraints. arXiv preprint arXiv:1802.02497.

Schrijver, A. 1998. Theory of linear and integer programming. John Wiley \& Sons.

Thanh, B. L.; Ruggieri, S.; and Turini, F. 2011. $k$-NN as an implementation of situation testing for discrimination discovery and prevention. In Proceedings of the 17th ACM SIGKDD International Conference on Knowledge Discovery and Data Mining, San Diego, CA, USA, August 21-24, 2011, 502-510.

Vaidya, P. M. 1989. Speeding-up linear programming using fast matrix multiplication (extended abstract). In 30th Annual Symposium on Foundations of Computer Science, Research Triangle Park, North Carolina, USA, 30 October - 1 November 1989, 332-337.

von Luxburg, U. 2006. A tutorial on spectral clustering. Technical Report TR-149, Max Planck Institute for Biological Cybernetics, Germany.

Wagstaff, K., and Cardie, C. 2000. Clustering with instancelevel constraints. In Proceedings of the Seventeenth National Conference on Artificial Intelligence and Twelfth Conference on on Innovative Applications of Artificial Intelligence, July 30 - August 3, 2000, Austin, Texas, USA., 1097-1192.

$\mathrm{Xu}, \mathrm{R}$., and Wunsch, D. C. 2005. Survey of clustering algorithms. IEEE Transactions on Neural Networks 16(3):645678. 